\title{
Evidence for a critical role of gene occlusion in cell fate restriction
}

Jedidiah Gaetz ${ }^{1}$, Kayla L Clift ${ }^{1}$, Croydon J Fernandes ${ }^{1}$, Frank Fuxiang Mao ${ }^{1,2}$, Jae Hyun Lee ${ }^{1}$, Li Zhang ${ }^{1}$, Samuel W Baker ${ }^{1}$, Timothy J Looney ${ }^{1}$, Kara M Foshay ${ }^{1}$, Wei-Hua Yu ${ }^{2}$, Andy Peng Xiang ${ }^{2}$, Bruce T Lahn ${ }^{1}$

${ }^{I}$ Department of Human Genetics, University of Chicago, Howard Hughes Medical Institute, Chicago, IL 60637, USA; ${ }^{2}$ Center for Stem Cell Biology and Tissue Engineering, Sun Yat-sen University, Guangzhou, Guangdong 510080, China

The progressive restriction of cell fate during lineage differentiation is a poorly understood phenomenon despite its ubiquity in multicellular organisms. We recently used a cell fusion assay to define a mode of epigenetic silencing that we termed "occlusion", wherein affected genes are silenced by cis-acting chromatin mechanisms irrespective of whether trans-acting transcriptional activators are present. We hypothesized that occlusion of lineage-inappropriate genes could contribute to cell fate restriction. Here, we test this hypothesis by introducing bacterial artificial chromosomes (BACs), which are devoid of chromatin modifications necessary for occlusion, into mouse fibroblasts. We found that BAC transgenes corresponding to occluded endogenous genes are expressed in most cases, whereas BAC transgenes corresponding to silent but non-occluded endogenous genes are not expressed. This indicates that the cellular milieu in trans supports the expression of most occluded genes in fibroblasts, and that the silent state of these genes is solely the consequence of occlusion in cis. For the BAC corresponding to the occluded myogenic master regulator Myf5, expression of the $M y f 5$ transgene on the BAC triggered fibroblasts to acquire a muscle-like phenotype. These results provide compelling evidence for a critical role of gene occlusion in cell fate restriction.

Keywords: cell fate restriction; occlusion; bacterial artificial chromosome

Cell Research (2012) 22:848-858. doi:10.1038/cr.2011.190; published online 29 November 2011

\section{Introduction}

In multicellular organisms, a single cell - the fertilized egg - undergoes proliferation and differentiation to give rise to all cell types in the body. During this process, the cells not only acquire the phenotypic identities of the lineages to which they have become committed but also lose the potential for all other lineages $[1,2]$. The latter phenomenon, known as cell fate restriction, is manifested as the general absence of dedifferentiation or transdifferentiation under physiological conditions. As yet, little is known about what underlies cell fate restriction. While the literature suggests the involvement of both transcription factors acting in trans and chromatin components acting in cis [3-8], the nature and extent by which either trans or cis mechanism contributes to cell fate restriction

\footnotetext{
Correspondence: Bruce T Lahn

E-mail: blahn@bsd.uchicago.edu

Received 11 September 2011; revised 20 September 2011; accepted 19 October 2011; published online 29 November 2011
}

remain poorly understood. In particular, although many studies have documented extensive chromatin changes during differentiation $[6,8]$, it is not clear whether these cis changes are themselves the primary cause of cell fate restriction, or whether they are merely the downstream effect of trans mechanisms. Indeed, there is a dearth of experimental approaches for testing whether gene silencing is due to cis or trans mechanism in various developmental and physiological contexts.

We recently developed a cell fusion assay to assess whether the primary causal mechanism of gene silencing lies in trans or cis [9, 10]. Specifically, the assay reveals that the transcriptional potential of a gene can exist in one of two states: competent or occluded. A competent gene is responsive to trans-acting factors in the cellular milieu, such that it is active when appropriate transcriptional activators are present, though it can also be silent when activators are absent or repressors are present. In contrast, an occluded gene is blocked by cis-acting, chromatin-based mechanisms from responding to transacting factors, such that it remains silent irrespective of 
whether transcriptional activators are present in the cellular milieu. While expressed genes are obviously competent, the cell fusion assay reveals that silent genes can be further divided into those that are still competent and those that are occluded (we will hereon refer to genes that are silent but still competent as activatable genes). Activatable genes are silenced by trans mechanisms (i.e., absence of transcriptional activators or presence of repressors), and can potentially be activated again when the cellular milieu changes. In contrast, occluded genes are silenced by cis mechanisms and cannot be activated even in the presence of transcriptional activators.

Our definition of "occlusion" bears an important distinction from the commonly used term "epigenetic silencing". In the literature, a gene is often said to be epigenetically silenced when its silent state is associated with certain chromatin marks. However, it is typically not clear whether these chromatin marks are the cause or effect for silencing. In contrast, the term "occlusion" refers only to the type of silencing where cis-acting chromatin mechanisms are the primary causal agent of the silent state irrespective of whether transcriptional activators for the gene are present in the cell $[9,10]$.

The chromatin-based mechanisms causally responsible for the occlusion of genes are not known, but there are candidates, including any of the numerous covalent histone modifications, DNA methylation, binding of repressive complexes, such as Polycomb, subnuclear localization, or other molecular processes and factors that are currently unknown.

We hypothesized that the occlusion of lineage-inappropriate genes might contribute to the restriction of cell fate $[9,10]$. Specifically, we proposed that as cells commit to a particular lineage, some of the key genes that specify alternative lineages are occluded, such that these cells lose their ability to express these genes. Implicit to the hypothesis is the assumption that some of the lineageinappropriate genes subjected to occlusion are present in a cellular milieu that is supportive of their expression, such that they would be expressed if it were not for their occluded state [10].

We decided to test this hypothesis by introducing bacterial artificial chromosomes (BACs) into mouse tail fibroblasts (these cells are designated 129TF). BACs are typically large enough to accommodate all or most cis-regulatory regions of a gene, and the lack of chromatin modifications ensures that BAC transgenes are not occluded when first entering the cell. As such, the expression status of the BAC transgenes should provide a readout of the trans-acting milieu of the cell. Active expression of a BAC transgene indicates a transcriptionally supportive milieu for that gene (i.e., the cell contains transcriptional activators for the gene), whereas the lack of expression indicates a transcriptionally non-supportive milieu (i.e., the cell lacks activators or contains repressors for the gene). We show here that BAC transgenes corresponding to occluded endogenous genes are frequently expressed, whereas BAC transgenes corresponding to activatable endogenous genes are not expressed. This means that the cellular milieu in trans is actually supportive of the expression of most occluded genes (i.e., transcriptional activators for these genes are present in the cell), and the only reason for their silent state is occlusion in cis. We further show that when the BAC corresponding to the occluded myogenic master regulator Myf5 was introduced into fibroblasts, expression of the Myf5 transgene on the BAC triggered a profound change of fate identity in these cells towards a muscle-like phenotype, indicating that occlusion of endogenous $M y f 5$ is essential for preventing fibroblasts from activating muscle programs. These findings reveal a critical role of occlusion in safeguarding somatic cell fate.

\section{Results}

Identification of occluded and activatable genes for BAC transgene analysis

We have been systematically mapping occluded and activatable genes in $129 \mathrm{TF}$ by fusing them to a variety of mouse and rat cell types, including mouse myoblasts (C2C12), rat myoblasts (L6), rat cardiomyoblasts (H9), rat osteosarcoma cells (UMR), and rat chondrocytes (IRC) (manuscript submitted). From the occluded and activatable genes that we identified in these fusions, 10 genes from each category were chosen for the BAC transgene analysis. We gave priority to genes involved in myogenesis because this is a well-studied system and was the focus of our recently published studies on occlusion [9, $11]$, but we also chose a number of genes randomly.

We first confirmed the occluded or activatable status of these genes in 129TF using the "RT-PCR-Seq" protocol. RT-PCR primers were designed to amplify a gene of interest from both $129 \mathrm{TF}$ and its fusion partner, flanking nucleotide sites that differ between the two cell types. RT-PCR product from fused cells was then sequenced, and the allele composition at these nucleotide sites was used to assess whether the gene was expressed from one or both of the two genomes in fused cells. For activatable genes in 129TF, RT-PCR showed a lack of expression in $129 \mathrm{TF}$ and robust expression in its fusion partner prior to fusion. After fusion, expression in fused cells came from both the 129TF genome and the fusion partner's genome, indicating that the 129TF copies of these genes were activated upon fusion (Figure 1A). For occluded genes in 
$129 \mathrm{TF}$, there is a lack of expression in $129 \mathrm{TF}$, but robust expression in its fusion partner prior to fusion, just like activatable genes. However, after fusion, expression in fused cells came only from 129TF's fusion partner and not from the $129 \mathrm{TF}$ genome, indicating that the $129 \mathrm{TF}$ copies of these genes failed to turn on in fused cells even though their orthologous copies in the fusion partner's genome were expressed (Figure 1B). We also included Myf6 and Chrnd in the BAC transgene analysis. The occlusion status of these two genes in 129TF is unknown. However, in other mouse fibroblast lines that we examined, Myf6 is consistently occluded while Chrnd is activatable ([9]; unpublished data). We therefore assumed that the same is true in $129 \mathrm{TF}$.

In total, 11 occluded and 11 activatable genes were subjected to the BAC transgene analysis. We obtained rat BACs corresponding to these genes and transfected them into 129TF (Supplementary information, Table S1). We used rat (as opposed to mouse) BACs because this allowed us to easily distinguish transgene expression from endogenous gene expression by exploiting sequence divergence between rat and mouse orthologs, while also minimizing potential interspecies genetic incompatibility given the close evolutionary relationship between mouse and rat. The BAC inserts ranged in size from 173 to 252 $\mathrm{kb}$, and for each gene, the corresponding BAC carried the entire transcriptional unit plus a minimum of $40 \mathrm{~kb}$ upstream and $27 \mathrm{~kb}$ downstream sequences (Supplementary information, Table S1). The great majority of BACs also contained additional genes that flanked the genes of interest. It is therefore likely that most, if not all, of the regulatory sequences in the genes of interest are present on the BACs. For ease of description, BAC transgenes corresponding to activatable endogenous genes will be referred to as "a-transgenes", whereas BAC transgenes corresponding to occluded endogenous genes will be called "o-transgenes."

We modified each BAC by inserting a neomycinselectable cassette into the vector backbone, transfected it into $129 \mathrm{TF}$ cells, and selected for stable BAC integration using neomycin. For each BAC, we chose up to three neomycin-resistant clones and performed RT-PCR with mouse/rat common primers for the genes of interest, followed by sequencing of RT-PCR product to examine whether the expression, if any, came from the rat BAC transgene or from the endogenous mouse gene.

\section{$B A C$ transgenes corresponding to activatable endog- enous genes are silent}

Because the silent state of the activatable genes is supposedly due to the presence of a transcriptionally non-supportive milieu acting in trans, we expected that when an a-transgene is introduced into the same milieu, it should behave identically as the endogenous activatable gene, meaning that it should also be silent. This is indeed our observation. In the 129TF clones carrying a-transgenes, there is no detectable RT-PCR product for all but one of the genes tested (Figure 2A). For Slc13A5, only one of the three clones that we initially examined had expression, suggesting that expression in this clone might be due to the effect of integration site. Consistent with this, when three additional clones containing the Slc13A5 BAC were examined, no Slc13A5 expression was detected in any of them (Figure 2A). Given that RTPCR primers were designed to equally amplify both the $\mathrm{BAC}$ transgenes and the endogenous genes, and given that these primers were validated on mouse and rat control cell types, the lack of RT-PCR product indicates that a-transgenes and their endogenous counterparts are both silent.

When several of the rat BACs carrying a-transgenes were introduced into $\mathrm{C} 2 \mathrm{C} 12$ mouse myoblasts where the corresponding mouse genes were already expressed, both mouse and rat copies of the genes were expressed in every case, as determined by sequencing of RT-PCR products (data not shown). This indicates that the lack of expression of a-transgenes in 129TF is not due to technical artifacts, such as the BACs not carrying regulatory sequences required for expression, genetic incompatibility between species, or de novo silencing of these BACs after transfection. Taken together, these data confirm that activatable genes are indeed silent due to trans-acting mechanisms.

\section{$B A C$ transgenes corresponding to occluded endogenous} genes are robustly expressed in most cases

Our expectations for o-transgenes were more complex. By definition, occluded genes are blocked by cisacting chromatin mechanisms from responding to transacting factors, such that they remain silent irrespective of whether the cellular milieu is supportive or non-supportive of their expression. If the milieu is indeed supportive of the expression of an occluded gene, the corresponding BAC transgene is expected to be expressed, but if the milieu is not supportive, the BAC transgene should be silent. Of the $11 \mathrm{o}$-transgenes introduced into $129 \mathrm{TF}, 10$ produced robust RT-PCR products, and for most of them, all clones showed positive expression (Figure 2B). This is in sharp contrast to the behavior of the a-transgenes (compare Figure 2A with 2B). Importantly, sequencing of the RT-PCR products revealed that expression came strictly from the BAC transgenes and not from their endogenous occluded counterparts (Figure 2B). It is important to note that the two a-transgenes that are silent in 

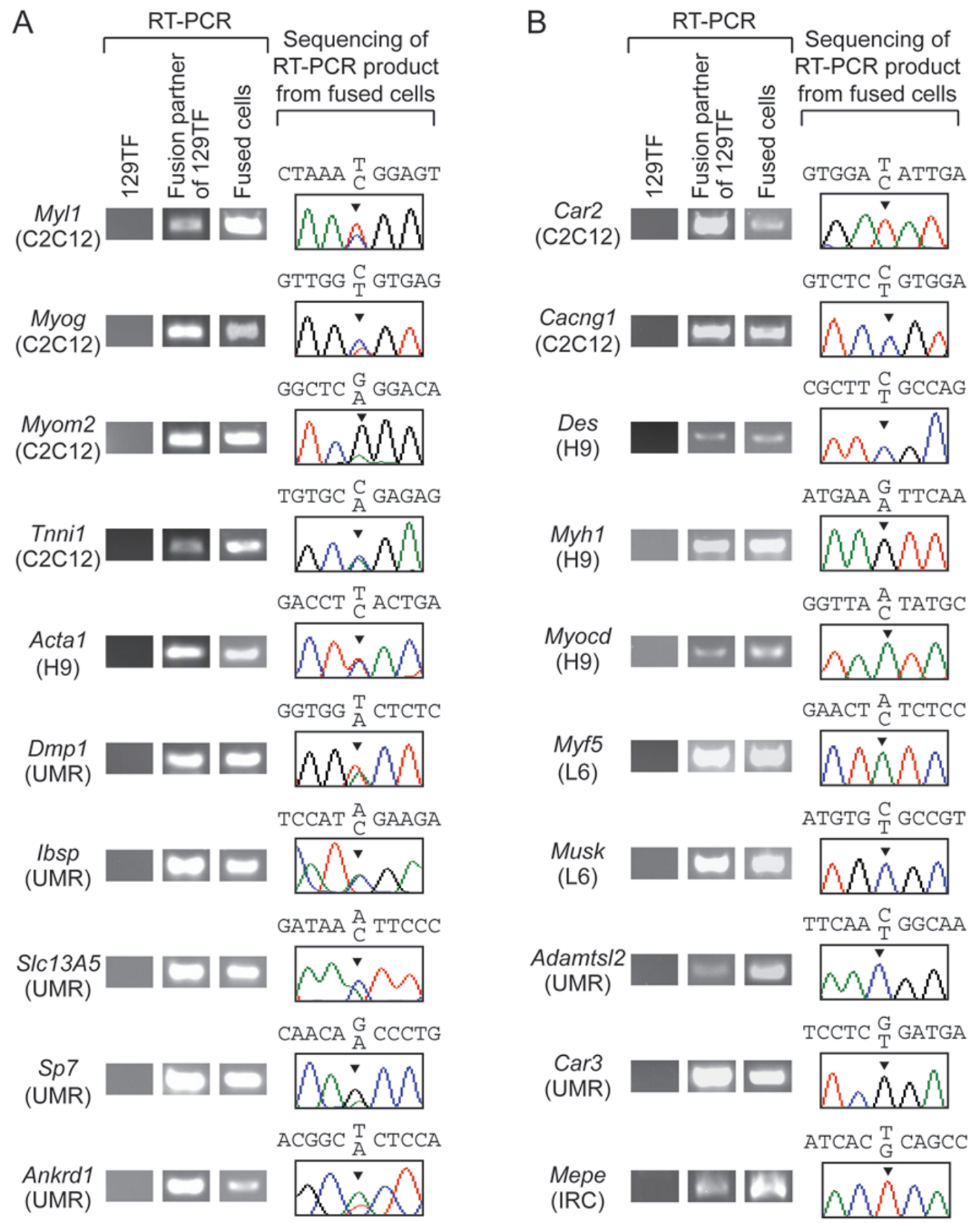

Figure 1 Assessing the activatable or occluded status of genes in 129TF. For each gene, the cell type used to fuse with $129 \mathrm{TF}$ in order to assess its activatable/occluded status in 129TF is indicated in parentheses below the gene name. Gel images are results of RT-PCR performed on 129TF, its fusion partner, and fused cells between the two. RT-PCR products from fused cells were sequenced, and the chromatograms corresponding to nucleotide sites that differ between 129TF and its fusion partner are shown. The sequence identities of these sites are given above the chromatograms (along with surrounding sequences), with the bottom and top alleles corresponding to 129TF and its fusion partner, respectively. Arrow heads indicate peaks of these sites in the chromatograms. (A) Activatable genes in 129TF. In fused cells, these genes show expression from both 129TF and its fusion partner. (B) Occluded genes in 129TF. In fused cells, these genes show no expression from 129TF and only expression from its fusion partner. 


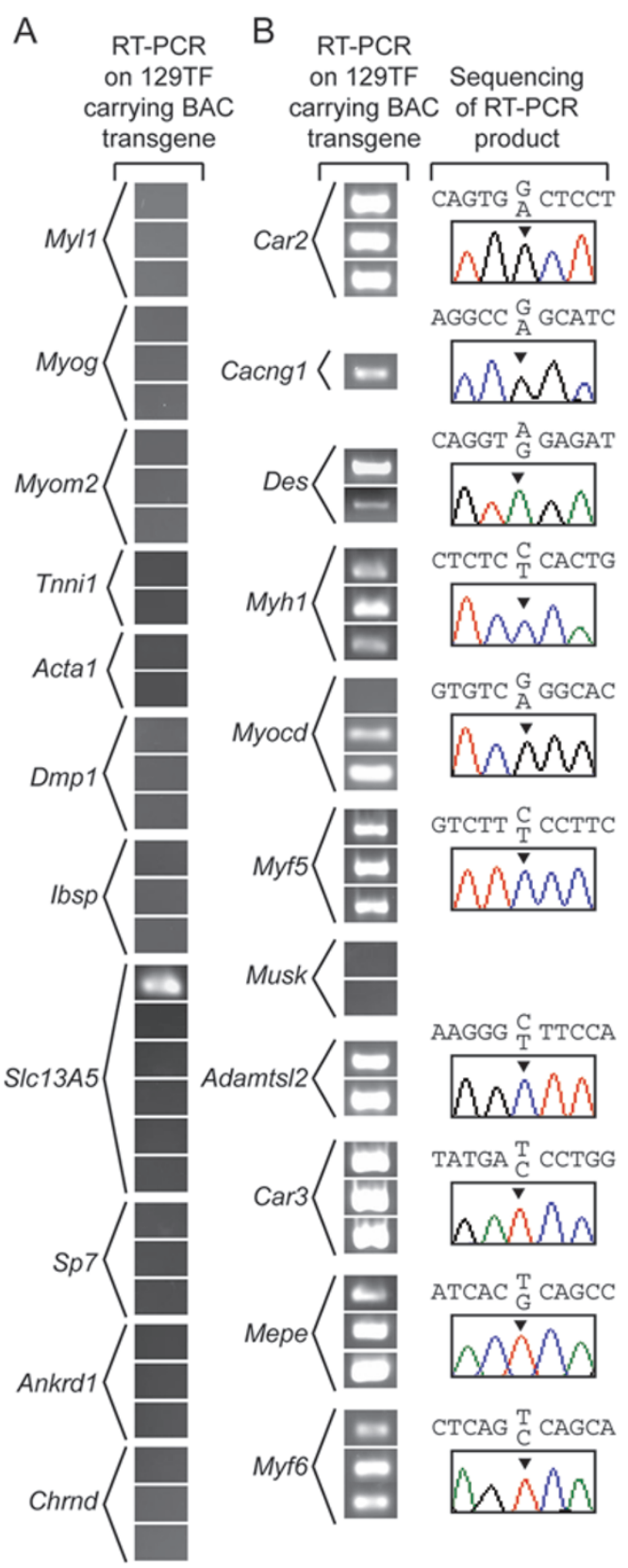

Figure 2 Expression status of BAC transgenes in 129TF. For each gene, RT-PCR was performed on all the 129TF clones carrying the corresponding BAC. (A) Lack of BAC transgene expression and endogenous gene expression in most 129TF clones carrying a-transgenes. (B) Robust BAC transgene expression in most 129TF clones carrying o-transgenes. For each o-transgene, RT-PCR products from all the clones were sequenced and a representative chromatogram corresponding to a nucleotide site that differs between the BAC transgene and the endogenous gene is shown. The sequence identities of such sites are given above the chromatograms (along with surrounding sequences), with the bottom and top alleles corresponding to the endogenous gene and the transgene, respectively. Arrow heads indicate peaks of these sites in the chromatograms. The chromatograms show that only the BAC transgenes and not the endogenous genes are expressed from the 129TF clones carrying o-transgenes.
129TF (Dmpl and Ibsp) and the one o-transgene that is active in 129TF (Mepe), all reside within the same BAC, arguing that the expression status of the transgenes is not dictated by some artifactual feature across the BAC. The sharply opposing behavior of the two categories of transgenes is statistically highly significant $\left(P<5 \times 10^{-14}\right.$ by Fisher's exact test performed at the level of clones or $P<$ $2 \times 10^{-5}$ when the test is performed at the level of genes). We were in fact quite surprised that the great majority otransgenes produced robust expression. This argues that due to occlusion in cis solely, the endogenous genes are kept silent despite the presence of a cellular milieu that contains all the requisite factors in trans to turn them on.

Occlusion of Myf5 is essential in preventing fibroblasts from acquiring a muscle-like phenotype

To further examine the role of gene occlusion in cell fate restriction, we carried out detailed analyses of three of the genes, Myf5, Myf6, and Myog, used in the BAC analysis. All three are master regulators of skeletal myogenesis, as evidenced by the fact that their forced expression in certain non-muscle cell types leads to the manifestation of a muscle-like phenotype, such as the formation of myotubes [12-15]. Additionally, evidence suggests that Myf5 and Myf6 act upstream of Myog during myogenesis [16]. While all three genes are silent in 129TF, Myf5 and Myf6 are occluded, whereas Myog is activatable (Figure 1). Myf5 and Myf6 are adjacent in the genome and are present on a single BAC. We will refer to this rat BAC as the rMyf5/6 BAC and the BAC carrying rat Myog as the rMyog BAC. In all 129TF clones carrying the rMyf5/6 BAC, both Myf5 and Myf6 transgenes are robustly expressed, while the endogenous copies of $M y f 5$ and Myf6 are silent (Figure 2B). In contrast, in all the $129 \mathrm{TF}$ clones carrying the rMyog BAC, the Myog transgene is silent just like the endogenous gene (Figure 2A).

Strikingly, myotubes were observed in the $129 \mathrm{TF}$ clones carrying the rMyf5/6 BAC (Figure 3A), indicating that the expression of the Myf5 and/or Myf6 transgenes have activated the myogenesis program in $129 \mathrm{TF}$. RTPCR confirmed that many muscle genes were indeed turned on in these cells (Figure 3B). Importantly, none of these downstream muscle genes are known to be occluded themselves, consistent with occluded genes remaining silent despite the presence of an activating cellular milieu. One of the genes activated in 129TF carrying the rMyf5/6 BAC is Myog, which is consistent with the known activatable status of this gene and the fact that Myog is thought to be functionally downstream of Myf5 [16]. Thus, the introduction of the rMyf5/6 BAC alone into $129 \mathrm{TF}$ has triggered fibroblasts to acquire a muscle- 
A
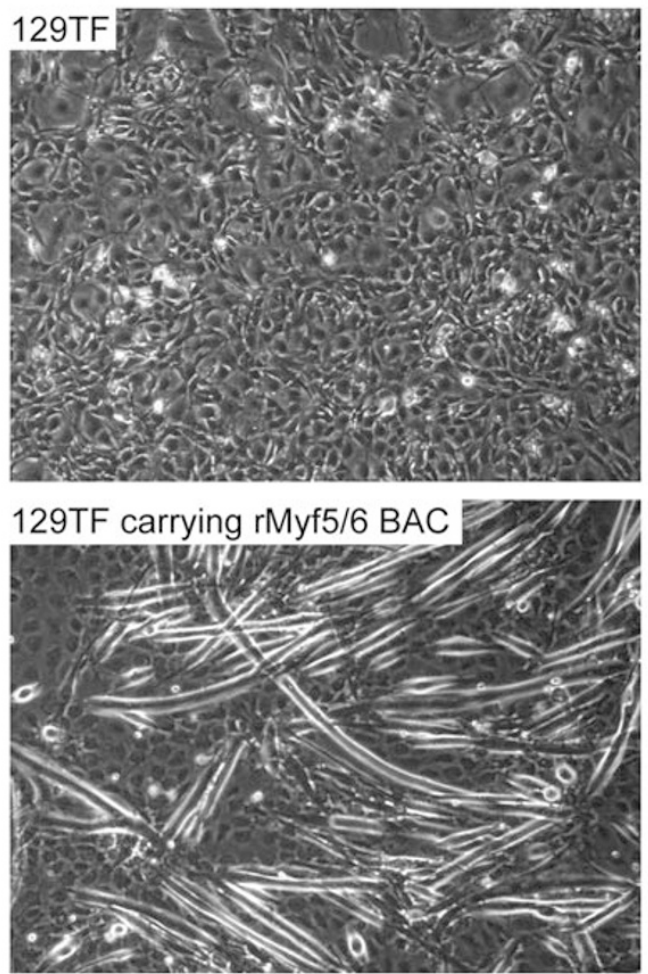

B

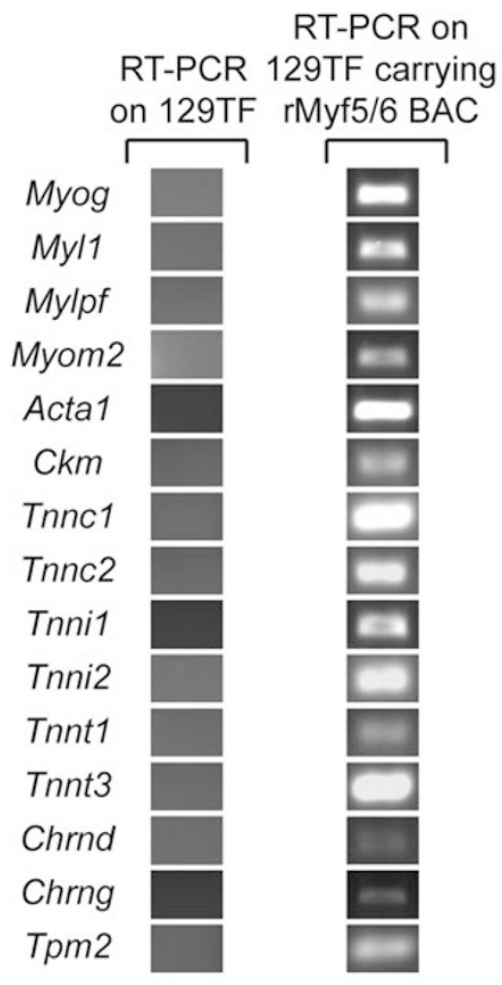

Figure 3 Acquisition of a muscle-like phenotype by 129TF carrying the rMyf5/6 BAC. (A) Phase-contrast microscopy images showing fibroblast morphology of regular 129TF and myotube morphology of 129TF carrying the rMyf5/6 BAC. (B) RT-PCR results showing that muscle-related genes are silent in normal 129TF and become activated in 129TF carrying the rMyf5/6 BAC.

like phenotype.

A simple interpretation of the robust expression of Myf5 and Myf6 transgenes in 129TF is that the cellular milieu of $129 \mathrm{TF}$ contains transcriptional activators for these two genes. However, there can be a more complex scenario, whereby, the $129 \mathrm{TF}$ milieu does not support the expression of one or both genes, but these genes engage in positive auto- and/or cross-regulation such that very low levels of initial background expression from the transgenes get amplified over time. To examine this possibility, we obtained a mouse BAC containing both $M y f 5$ and Myfo (hereon referred to as the mMyf5/6 BAC; see Supplementary information, Table S1), and knocked out both genes by replacing their first exons with a knockin cassette. When transfected into $129 \mathrm{TF}$, robust expression of the knockin cassette was observed from both loci based on RT-PCR (data not shown). This argues that 129TF contains transcription factors capable of driving the expression of both $M y f 5$ and $M y f 6$. We also knocked out Myf5 or Myf6 individually from the mMyf5/6 BAC and transfected the resulting BACs into 129TF. Myotube formation was observed only in the Myf6 knockout BAC (data not shown), indicating that the expression of the $M y f 5$ (but not $M y f 6$ ) transgene alone can lead to myotube formation in 129TF.

BAC transgenes introduced into cells in early development behave similarly as endogenous genes

One inference from the above data is that the establishment of occlusion during differentiation and its subsequent maintenance in differentiated cells likely involve distinct mechanisms, the former being present only transiently during differentiation, whereas the latter remains in effect indefinitely. This would explain the observation that when a BAC transgene is introduced into fibroblasts in which the endogenous counterpart is already occluded, the transgene does not undergo de novo occlusion even after extended culture. Accordingly, we predicted that if the rMyf5/6 BAC is introduced into cells at an early stage of development before the occlusion of the endogenous Myf5 and Myf6 is established, the Myf5 and Myf6 transgenes should become occluded in fibroblasts just like the endogenous genes.

To test this, we performed pronuclear injection of 
the rMyf5/6 BAC into one-cell mouse embryos to obtain transgenic animals carrying the BAC. We then derived tail fibroblasts from the animals, referred to as rMyf5/6(TG) fibroblasts. RT-PCR showed that both the Myf5 transgene and the endogenous Myf5 are silent in these fibroblasts, and the same is true for Myf6 (Figure 4A). Furthermore, RT-PCR showed that the tissue expression patterns of Myf5 and Myf6 transgenes are the same as the endogenous genes, with strong expression in skeletal muscle for both genes and no detectable expression in a panel of other tissues (Figure 4A). Sequencing of the RT-PCR products from rMyf5/6(TG) muscle showed that both BAC transgene copies and endogenous copies of $M y f 5$ and $M y f 6$ are expressed (Figure 4A). To examine whether the BAC transgenes are occluded in rMyf5/6(TG) fibroblasts, we fused these cells with C2C12 mouse myoblasts and performed RT-PCR using mouse/rat common primers. Sequencing of RT-PCR products showed that for both Myf5 and Myf6, only the mouse ortholog from the $\mathrm{C} 2 \mathrm{C} 12$ genome is expressed in fused cells, indicating that the Myf5 and Myf6 BAC transgene is indeed occluded in rMyf5/6(TG) fibroblasts (Figure 4B).

For comparison, we also used pronuclear injection to produce transgenic animals carrying the rMyog BAC, and derived tail fibroblasts from transgenic animals. In these $\mathrm{rMyog}(\mathrm{TG})$ fibroblasts, RT-PCR revealed no expression from either the Myog transgene or the endogenous Myog. Furthermore, tissue expression patterns of the Myog transgene is the same as the endogenous gene, with prominent expression in skeletal muscle and no detectable expression in other tissues (Figure 4A). Sequencing of the RT-PCR product from muscle showed that both the BAC transgene copy and the endogenous copy of Myog are expressed (Figure 4A). To interrogate the occlusion status of the Myog transgene in rMyog(TG) fibroblasts, we fused these cells to $\mathrm{C} 2 \mathrm{C} 12$, and performed RT-PCR on Myog using mouse/rat common primers followed by sequencing of RT-PCR product. This revealed that the Myog BAC transgene in $\mathrm{rMyog}(\mathrm{TG})$ fibroblasts is activatable, as it turned on in fused cells (Figure 4B).

Thus, the BAC transgene copies of Myf5, Myf6 and Myog behave just like their endogenous counterparts in terms of expression patterns and occlusion status when introduced into cells at a very early developmental stage (i.e., one-cell embryo). However, when introduced acutely into terminally differentiated fibroblasts, the Myog transgene is silent just as its silent (and activatable) endogenous counterpart, whereas the Myf5 and Myf6 transgenes are actively expressed in contrast to their silent (and occluded) endogenous counterparts.

Taken together, these observations argue that the occlusion of Myf5 and Myf6 is established early during development and subsequently maintained in differ-
A

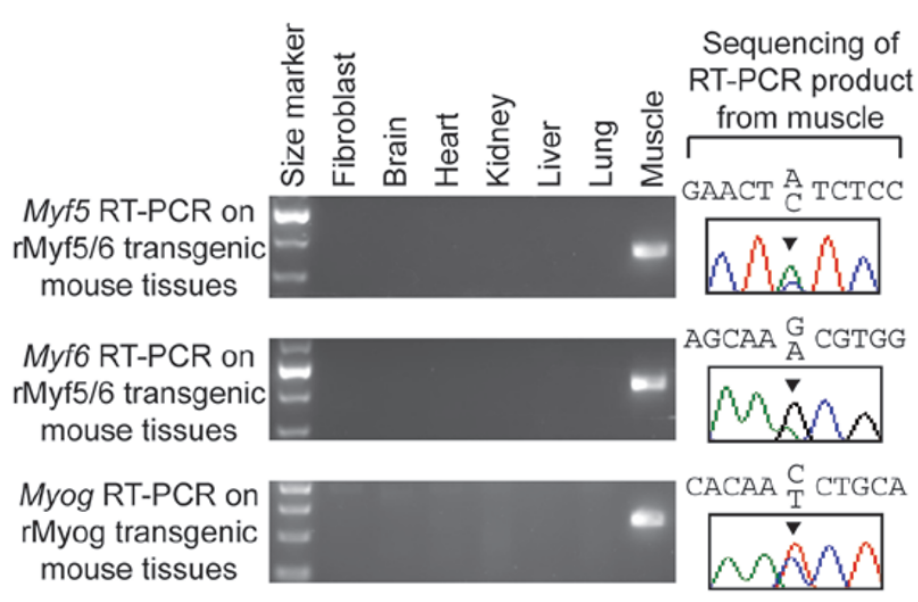

B

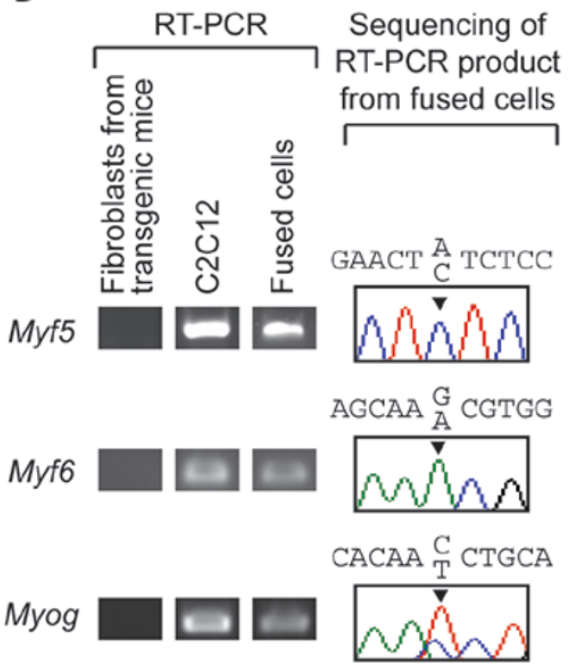

Figure 4 Expression pattern and occlusion status of Myf5, Myf6, and Myog transgenes in transgenic mice. (A) Comparable expression pattern of transgene copies and endogenous copies of Myf5, Myf6, and Myog in multiple tissues and tail fibroblasts. RT-PCR using mouse/rat common primers revealed robust expression only in the skeletal muscle for Myf5, Myf6, and Myog. For each gene, sequencing of the RT-PCR product from muscle revealed expression from both the transgene and the endogenous gene. (B) Different occlusion status of the Myf5 and Myf6 transgenes versus the Myog transgene. In fused cells between fibroblasts derived from transgenic mice and $\mathrm{C} 2 \mathrm{C} 12$, the Myf5 and Myf6 transgenes are silent, indicating that they are occluded. In contrast, the Myog transgene is expressed, indicating that it is activatable. 
entiated cells, and that the occlusion of these genes by cis-acting mechanisms is absolutely required for their stable silencing in a cellular milieu that would otherwise activate them and cause cells to acquire a muscle-like phenotype. In contrast, Myog does not undergo occlusion in fibroblasts, and its silent state in these cells is due to trans-acting mechanisms (i.e., absence of transcriptional activators). The fact that $\mathrm{BAC}$ transgenes in transgenic animals behave in the same way as their endogenous counterparts also indicates that these BACs contain all the necessary regulatory sequences for their proper expression (and proper occlusion in the case of the rMyf5/6 $\mathrm{BAC})$.

\section{Discussion}

To understand cell fate restriction is to understand how gene expression patterns characteristic of individual cell types are stably maintained. While both trans-acting mechanisms involving diffusible factors and cis-acting mechanisms involving chromatin modifications have been implicated [3-7], definitive data on the nature and extent by which either trans or cis mechanisms contribute to cell type-specific gene expression patterns are lacking. The present study provides compelling evidence that gene occlusion - a mode of gene silencing mediated by cis mechanisms - plays a prominent and critical role in cell fate restriction.

By using a BAC transgene approach, we make the surprising observation that for the majority of occluded genes, the transcriptional milieu in trans is actually supportive of their expression, meaning that the cell contains transcriptional activators for most occluded genes. Our study further shows that without the occlusion of a single gene, $M y f 5$, fibroblasts would acquire a muscle-like phenotype. Our recent effort to systematically map occluded genes showed that the number of occluded genes in a typical somatic cell type could be in the thousands (manuscript submitted). The present study argues that most of these genes would have been expressed if it were not for their occluded status. We therefore conclude that occlusion has an essential function in safeguarding somatic cell fate by preventing the expression of a large number of lineage-inappropriate genes.

The lack of expression of the single o-transgene, Musk, may be due to the absence of a transcriptionally supportive milieu, suggesting that the presence of such a milieu is not completely universal for all occluded genes, even if it is the case for the majority. However, an alternative explanation is that the lack of expression of this outlier gene is due to the fact that it is the largest gene included in this study, and the relatively small amount of flanking genomic sequence included in the BAC may have resulted in the loss of a necessary positive regulatory element far from the gene.

The importance of occlusion in cell fate restriction, as reported here, would seem incompatible with the relative ease by which somatic cells can be dedifferentiated towards pluripotency by experimental manipulations, such as SCNT into oocytes [17, 18], or forced expression of the iPSC factors $[19,20]$. In a submitted manuscript, we resolved this paradox by showing that pluripotent stem cells, unlike somatic cells, have the unique capacity for global deocclusion (i.e., erasure of occlusion on a wholegenome scale). We therefore argue that somatic cells can be experimentally reprogrammed to pluripotency either by tapping into the existing deocclusion capacity of pluripotent cells (i.e., SCNT into oocytes) or by recapitulating key aspects of the deocclusion machinery (i.e., the use of defined factors to create iPSCs).

The fact that occluded genes in somatic cells do not turn on, even though transcriptional activators for these genes are often present in the cellular milieu, would seem inconsistent with reports that forced expression of certain transcription factors can drive one somatic cell type to transdifferentiate into another, such as fibroblasts to myotube-like cells [21], to hepatocytes-like cells [22, 23], to cardiomyocyte-like cells [24, 25], to macrophagelike cells [26], and to neuron-like cells [27]. Indeed, one influential school of thought holds that the differentiated state is continuously and dynamically regulated by the balance of transcription factors, and a tip in such balance could lead to transdifferentiation [3, 4, 28, 29]. We offer two possible explanations for the apparent contradiction between occlusion and transdifferentiation. First, previous claims of transdifferentiation by forced expression of transcription factors often examined only a limited number of cell type-specific markers. It is hence possible that the downstream genes activated by these transcription factors are mostly activatable genes and many - if not all - occluded genes are still silent due to occlusion. If this is the case, then some of the claims of transdifferentiation might not entail the complete conversion of one cell type to another; but rather, they might represent a partial (and incomplete) acquisition of gene expression patterns characteristic of the second cell type, a situation that is perhaps better called "ectopic differentiation" rather than true transdifferentiation [10]. This is supported by our observation that when the $\mathrm{rMyf5/6} \mathrm{BAC}$ is transfected into fibroblasts, although the Myf5 BAC transgene is expressed and this expression induces the cells to assume a muscle-like phenotype, these cells do not express the endogenous Myf5 (which is occluded) or other occluded genes examined. Likewise, forced overexpression 
of the related bHLH transcription factor MyoDl as a transgene in $129 \mathrm{TF}$ was also able to induce muscle-like phenotype without activation of endogenous Myf5 (unpublished data). Thus, whereas these cells do show some resemblance to muscle in terms of gene expression and cellular physiology, they are different from naturally derived muscle cells (e.g., they do not express endogenous Myf5). Indeed, for the "transdifferentiation" studies cited above, the endogenous genes in $129 \mathrm{TF}$ corresponding to many of the overexpressed transgenes were found to be occluded (manuscript submitted), prompting us to speculate that the endogenous copies of these genes (as well as other occluded genes) might actually not be activated in these transdifferentiation studies. Notwithstanding the above discussion, we also propose an alternative explanation, namely, it is also possible that the overexpression of certain transcription factors far beyond their physiological levels might indeed be able to activate some occluded downstream genes and might even permanently erase occlusion. This is plausible, given the fact that many transdifferentiation experiments use strong promoters to drive exogenously introduced transcription factor genes, which can result in supraphysiological expression levels.

Importantly, our results suggest that the transcriptional environment in trans in different cell types are perhaps much more similar than previously appreciated, and that the differential expression of genes between different cell types is, to a large extent, the result of differential occlusion in cis rather than the presence of different factors in trans. Indeed, we propose that at the most fundamental level, the identities of individual cell types are defined largely by the occlusion status of their genomes.

\section{Materials and Methods}

\section{Ethics statement}

All animal work was conducted according to relevant national and international guidelines.

\section{Cell culture}

The $\mathrm{C} 2 \mathrm{C} 12$ mouse myoblasts of $\mathrm{C} 3 \mathrm{H}$ strain background were obtained from ATCC (Cat\# CRL-1772), and two clonal populations, named $\mathrm{C} 2 \mathrm{C} 12-\mathrm{GPc} 60$ and $\mathrm{C} 2 \mathrm{C} 12-\mathrm{RHc} 2$, were derived from cells transduced with a lentiviral vector containing EGFP driven by the human EF1A promoter and puromycin resistance, and cells transduced with a lentiviral vector containing dTomato [30] driven by the human EF1A promoter and hygromycin resistance. Tail fibroblasts derived from rMyf5/6(TG) and $\mathrm{rMyog}(\mathrm{TG})$ mice were transduced with a lentiviral vector containing dTomato driven by the human EF1A promoter and hygromycin resistance. Lentiviral vectors are as described previously [31]. The origins of the rat cells are as follows: IRC (full name: IRC-RHc17) from IRC chondrocytes [32] (gift from Walter Horton), L6 (full name: L6-RHc6) from L6 myoblasts (ATCC, Cat\# CRL-1458), H9 (full name: H9-RHcA10) from H9c2(2-1) cardiomyoblasts (ATCC, Cat\# CRL-1446), and UMR (full name: UMR-RHc7) from UMR106 osteosarcoma (ATCC, Cat\# CRL1661). Each of these rat cell types was a clonal population derived from cells transduced with a lentiviral vector carrying constitutively expressed DsRed-Express2 [33] or dTomato driven by the human EF1A promoter and hygromycin resistance as described previously. They were cultured under conditions as published or recommended by the vendor. The lentivirus-transduced cells used in this study are available through Cyagen Biosciences. Fusions between tail fibroblasts from BAC transgenic mice and $\mathrm{C} 2 \mathrm{C} 12-\mathrm{GPc} 60$, as well as between $129 \mathrm{TF}$ and $\mathrm{C} 2 \mathrm{C} 12-\mathrm{RHc} 2$, were carried out as follows. $\mathrm{C} 2 \mathrm{C} 12$ cells were first cultured under low-serum conditions. The two cell types were then plated together for $6 \mathrm{~h}$, followed by overlaying a solution of $37{ }^{\circ} \mathrm{C}$ PEG $1500 \mathrm{MW}(50 \% \mathrm{w} / \mathrm{w}$ in serum-free DMEM) for $1 \mathrm{~min}$. After removal of PEG, cells were washed three times with warm serumfree DMEM and allowed to recover for $4 \mathrm{~h}$ at $37^{\circ} \mathrm{C}$, followed by transition to media containing $1 \mu \mathrm{g} / \mathrm{ml}$ puromycin and $400 \mu \mathrm{g}$ / $\mathrm{ml}$ hygromycin to select for dual drug-resistant fused cells. Total RNA was harvested for cDNA preparation 5 days after fusion. All other fusions were performed following the same general protocol as follows. Cells were plated together for at least $2 \mathrm{~h}$ (or overnight). Prior to fusion, cells were washed with serum-free DMEM, and PEG $1500 \mathrm{MW}$ or $1000 \mathrm{MW}$ (50\% w/v in serum-free DMEM) was added for $1 \mathrm{~min}$. After removal of PEG, cells were washed three times with serum-free DMEM and allowed to recover for 2 h. Following this, cells were split to a lower density and plated in media containing both puromycin and hygromycin to select for double drug-resistant fused cells. Daily trypsinization aided in selection and purification of fused cells, with total RNA harvested between 6-7 days post fusion. Variations in this protocol included cell to cell ratio, cell density, and concentrations of puromycin and hygromycin, all of which were determined empirically for each fusion. L6 was differentiated under low-serum condition prior to fusion.

\section{RT-PCR-Seq}

Total RNA was purified from cell or tissue samples using the ZR RNA MiniPrep kit (Zymo Research) and treated with DNase I to remove contaminating genomic DNA. Reverse transcription was carried out using the SuperScript III First-Strand Synthesis System (Invitrogen) with random hexamer primers following vendor's instructions. PCR was performed in $20 \mu \mathrm{l}$ reactions and 35 cycles using Platinum Taq Hi-Fidelity (Invitrogen) following vendor's instructions. Each reaction used template cDNA converted from $2.5 \mathrm{ng}$ of total RNA. PCR products were treated with shrimp alkaline phosphatase and E. coli exonuclease I (US Biologicals), followed by an ABI BigDye Terminator reaction and sequencing using an ABI 3730xl DNA Analyzer (Applied Biosystems). Mouse/rat (or mouse/mouse) common primers were designed from primer-binding sequences that are identical between the two species (or between the two mouse strains being investigated), and which flank sequence differences between the two, without indels, based on cDNA sequence alignments. Whenever possible, primers were designed such that the resulting amplicon spanned one or more introns. This way, contaminating genomic DNA would not result in RT-PCR product of the right size. Mouse and rat cDNA sequences were based on RefSeq sequences retrieved from NCBI. Polymorphisms between mouse strains were identified from the 
Perlegen Mouse HapMap as published [34], or by genomic PCR and sequencing of PCR product. To perform genomic PCR and sequencing, primers were designed to amplify individual exons from genomic DNA plus at least 50 bp flanking sequences on either side, followed by sequencing of PCR products. Sequences of PCR primers are available upon request. All RT-PCR primers were validated based on their ability to amplify products from positive control cell types where the target genes were known to be expressed.

\section{$B A C$ preparation and transfection}

BACs of interest were purchased from the Children's Hospital Oakland Research Institute BACPAC Resources Center, and were modified to introduce a neomycin resistance cassette as described previously [35]. Gene replacement in the mMyf5/6 BAC was carried out using Red/ET recombination system (Gene Bridges) following vendor's instructions and as previously described [36]. For either $M y f 5$ or $M y f 6$, the ORF portion of the first exon plus two adjacent bases of the first intron were replaced with a knockin cassette [36]. Myf5 was replaced with a cassette consisting of dTomato, followed by IRES (internal ribosome entry site), GB3 (a bacterial promoter), and neo (neomycin resistance gene). Myf6 was replaced with a cassette consisting of eGFP, followed by IRES, GB3, and $b s d$ (blasticidin resistance gene). BACs were prepared using the PowerPrep HP Maxiprep kit (Marligen Biosciences), and transfected as described [37]. 129TF cells were plated at approximately $30 \%$ confluency in $30-\mathrm{mm}$ dishes the day before transfection, expanded to $10-\mathrm{cm}$ dishes 2 days after transfection, and maintained in $400 \mu \mathrm{g} / \mathrm{ml}$ neomycin (G418) selection medium 5 days after transfection. For isolation of stable BAC transfected clones, neomycin-resistant cells were trypsinized, and individual cells distributed into wells of 96-well plates using a FACSAria II Flow Cytometer (BD Biosciences). Clones were expanded in medium containing $400 \mu \mathrm{g} / \mathrm{ml}$ neomycin until subsequent analysis.

\section{Generation of BAC transgenic mice}

The production of transgenic mice and the derivation of tail fibroblasts from transgene-positive animals were performed by Cyagen Biosciences. Unmodified rMyf5/6 BAC and rMyog BAC were used to make transgenic mice by pronuclear injection of $\mathrm{BAC}$ DNA into one-cell embryos of FVB strain background following standard procedures.

\section{Acknowledgments}

We thank the following individuals for technical assistance and/ or critical discussions: Nicholas Heintz, Ralf Kittler, Patrick Reed, Ursula Storb, Alec Victorsen, and Kevin White. This work was funded in part by NIH postdoctoral fellowships F32HL922792 (to JG) and F32HD061205 (to KMF), the National Natural Science Foundation of China grant $(30971675,30928015)$ and the Key Scientific and Technological Projects of Guangdong Province grant 2007A032100003 (to APX); and the Chicago Biomedical Consortium with support from The Searle Funds at The Chicago Community Trust (to BTL).

\section{References}

1 Waddington $\mathrm{CH}$. Organisers and Genes. London: Cambridge
University Press, 1940.

2 Waddington $\mathrm{CH}$. The Strategy of the Genes. London: George Allen \& Unwin, 1957.

3 Blau HM, Baltimore D. Differentiation requires continuous regulation. J Cell Biol 1991; 112:781-783.

4 Graf T, Enver T. Forcing cells to change lineages. Nature 2009; 462:587-594.

5 Yamanaka S, Blau HM. Nuclear reprogramming to a pluripotent state by three approaches. Nature 2010; 465:704-712.

6 Reik W. Stability and flexibility of epigenetic gene regulation in mammalian development. Nature 2007; 447:425-432.

7 Goldberg AD, Allis CD, Bernstein E. Epigenetics: a landscape takes shape. Cell 2007; 128:635-638.

8 Hirabayashi Y, Gotoh Y. Epigenetic control of neural precursor cell fate during development. Nat Rev Neuroscience 2010; 11:377-388.

9 Lee JH, Bugarija B, Millan EJ, et al. Systematic identification of cis-silenced genes by trans complementation. Hum Mol Genet 2009; 18:835-846.

10 Lahn BT. The "occlusis" model of cell fate restriction. BioEssays 2010; 33:13-20.

11 Lee JH, Gaetz J, Bugarija B, et al. Chromatin analysis of occluded genes. Hum Mol Genet 2009; 18:2567-2574.

12 Braun T, Buschhausen-Denker G, Bober E, Tannich E, Arnold $\mathrm{HH}$. A novel human muscle factor related to but distinct from MyoD1 induces myogenic conversion in 10T1/2 fibroblasts. EMBO J 1989; 8:701-709.

13 Wright WE, Sassoon DA, Lin VK. Myogenin, a factor regulating myogenesis, has a domain homologous to MyoD. Cell 1989; 56:607-617.

14 Braun T, Bober E, Winter B, Rosenthal N, Arnold HH. Myf-6, a new member of the human gene family of myogenic determination factors: evidence for a gene cluster on chromosome 12. EMBO J 1990; 9:821-831.

15 Miner JH, Wold B. Herculin, a fourth member of the MyoD family of myogenic regulatory genes. Proc Natl Acad Sci USA 1990; 87:1089-1093.

16 Tapscott SJ. The circuitry of a master switch: MYOD and the regulation of skeletal muscle gene transcription. Development 2005; 132:2685-2695.

17 Gurdon JB. The developmental capacity of nuclei taken from intestinal epithelium cells of feeding tadpoles. J Embryol Exp Morphol 1962; 10:622-640.

18 Wilmut I, Schnieke AE, McWhir J, Kind AJ, Campbell KH. Viable offspring derived from fetal and adult mammalian cells. Nature 1997; 385:810-813.

19 Takahashi K, Yamanaka S. Induction of pluripotent stem cells from mouse embryonic and adult fibroblast cultures by defined factors. Cell 2006; 126:663-676.

20 Okita K, Ichisaka T, Yamanaka S. Generation of germlinecompetent induced pluripotent stem cells. Nature 2007; 448:313-317.

21 Davis RL, Weintraub H, Lassar AB. Expression of a single transfected cDNA converts fibroblasts to myoblasts. Cell 1987; 51:987-1000.

22 Huang P, He Z, Ji S, et al. Induction of functional hepatocytelike cells from mouse fibroblasts by defined factors. Nature 2011; 475:386-389.

23 Sekiya S, Suzuki A. Direct conversion of mouse fibroblasts 
to hepatocyte-like cells by defined factors. Nature 2011; 475:390-393.

24 Ieda M, Fu JD, Delgado-Olguin P, et al. Direct reprogramming of fibroblasts into functional cardiomyocytes by defined factors. Cell 2010; 142:375-386.

25 Efe JA, Hilcove S, Kim J, et al. Conversion of mouse fibroblasts into cardiomyocytes using a direct reprogramming strategy. Nat Cell Biol 2011; 13:215-222.

26 Feng R, Desbordes SC, Xie H, et al. PU.1 and C/EBPalpha/ beta convert fibroblasts into macrophage-like cells. Proc Natl Acad Sci USA 2008; 105:6057-6062.

27 Vierbuchen T, Ostermeier A, Pang ZP, Kokubu Y, Sudhof TC, Wernig M. Direct conversion of fibroblasts to functional neurons by defined factors. Nature 2010; 463:1035-1041.

28 Blau HM. How fixed is the differentiated state? Lessons from heterokaryons. Trends Genet 1989; 5:268-272.

29 Slack JM. Metaplasia and transdifferentiation: from pure biology to the clinic. Nat Rev Mol Cell Biol 2007; 8:369-378.

30 Shaner NC, Campbell RE, Steinbach PA, Giepmans BN, Palmer AE, Tsien RY. Improved monomeric red, orange and yellow fluorescent proteins derived from Discosoma sp. red fluorescent protein. Nat Biotechnol 2004; 22:1567-1572.
31 Qin JY, Zhang L, Clift KL, et al. Systematic comparison of constitutive promoters and the doxycycline-inducible promoter. PLoS One 2010; 5:e10611.

32 Horton WE Jr, Cleveland J, Rapp U, et al. An established rat cell line expressing chondrocyte properties. Exp Cell Res 1988; 178:457-468.

33 Strack RL, Strongin DE, Bhattacharyya D, et al. A noncytotoxic DsRed variant for whole-cell labeling. Nat Methods 2008; 5:955-957.

34 Frazer KA, Eskin E, Kang HM, et al. A sequence-based variation map of 8.27 million SNPs in inbred mouse strains. $\mathrm{Na}$ ture 2007; 448:1050-1053.

35 Wang Z, Engler P, Longacre A, Storb U. An efficient method for high-fidelity BAC/PAC retrofitting with a selectable marker for mammalian cell transfection. Genome Res 2001; 11:137-142.

36 Poser I, Sarov M, Hutchins JR, et al. BAC TransgeneOmics: a high-throughput method for exploration of protein function in mammals. Nat Methods 2008; 5:409-415.

37 Phelps SF, Illenye S, Heintz NH. Manipulating genes and gene copy number by bacterial artificial chromosomes transfection. Methods Mol Biol 2007; 383:153-163.

(Supplementary information is linked to the online version of the paper on the Cell Research website.) 